\title{
Obesity Prevention in Scotland: A Policy Analysis Using the ANGELO Framework
}

\author{
John D. Mooney ${ }^{a}$ b Ruth Jepson ${ }^{b} \quad$ John Frank ${ }^{b}$ Rosemary Geddes ${ }^{b}$ \\ aDepartment of Pharmacy, Health \& Well-Being, University of Sunderland, Health Sciences \\ Complex, City Campus, Sunderland, UK; ${ }^{b}$ Scottish Collaboration for Public Health Research \\ and Policy, Department of Public Health Sciences, University of Edinburgh, Edinburgh, UK
}

\section{Key Words}

Obesity · Prevention · Public policy · ANGELO framework · Obesity prevention · Scotland

\begin{abstract}
Background: The Scottish Government's 'Route-Map Action Plan' for obesity prevention sets out 62 potential intervention policies across all stages of the life course. We used the ANGELO Framework (Analysis Grid for Environments Linked to Obesity) to assess the appropriateness and likely impact of the balance of measures being proposed. Methods: Two assessors (JM \& $\mathrm{RJ}$ ) independently allocated a category for each intervention according to its domain (physical, economic, legislative or socio-cultural), scale (macro or micro) and predominant 'focus' (physical activity versus diet). A third assessor (RG) examined discordant allocations. Results: Across the four ANGELO domains, the distribution of interventions was skewed towards sociocultural measures (37.1\%) and the physical environment (33.1\%) with less emphasis on legislative or economic interventions ( $21.8 \%$ and $6.4 \%$ respectively). Distribution by both intervention scale and focus was more even with just over half of all policies (51.8\%) at the macro-level scale and just under half (46.7\%) having a dietary focus. Conclusion: The predominance of socio-culturally orientated interventions over their legislative and economic counterparts is at odds with the balance of international evidence on what would be most effective for obesity prevention. The ANGELO framework provides a useful tool for policy makers to monitor progress towards an appropriately balanced policy mix.
\end{abstract}

C 2015 S. Karger GmbH, Freiburg 
Mooney et al.: Obesity Prevention in Scotland: A Policy Analysis Using the ANGELO Framework

\section{Introduction}

Scotland has recently been confirmed as having one of the highest prevalence rates of adult obesity in the world [1]. It is therefore hardly surprising that there have been significant efforts in recent years to identify and develop effective population-wide interventions to stabilise and eventually reverse this worrying statistic. In response, the Scottish Government set out a diverse menu of potential interventions across all sectors of Government. Entitled 'A Route-Map towards a Healthy Weight' [2], its purpose was to set the direction of travel for the cultivation and development of both existing and new obesity prevention policies and programmes. The document recognises from the outset that the scale of the changes required 'cannot solely rely on individual behaviour changes to make the difference.' This acknowledgement is very much in accordance with the earlier Foresight Report [3], commissioned by the UK Government, which highlighted the environmental aetiology of the epidemic and hence the need for structural and legislative interventions and the avoidance of an overreliance on personal behaviour modification.

The Route-Map also acknowledged that, while there was much work that was already being done (the total investment in obesity prevention programmes and activities by the Scottish Government estimated to be in the range of GBP 200 millon between 2008 and 2011), the relevant initiatives and programmes had largely developed independently of each other without overall co-ordination [4]. As a first step in striving to improve the current combination of policy measures, the public health directorate within Scottish Government undertook a comprehensive audit of all relevant policies and programmes which were either currently active or in the planning stages. Publishing the findings of this audit in the Route-Map Action Plan [4], a total of 62 policy initiatives and programmes (which were underway or in the planning stages) from across all Scottish Government departments was found that could potentially contribute to obesity prevention. While the primary focus of many of these programmes might not have been to target population excess weight, the expansion in obesity's 'web of causation' means that many general health and environmental improvement measures have the potential to contribute to efforts aimed at tackling the epidemic.

At the same time as the Route-Map document was being developed, the Scottish Collaboration for Public Health Research and Policy (SCPHRP) had completed a review on the international evidence of obesity prevention, with a focus on working age adults [5]. Our approach in that review was first to identify a policy categorisation system that could be used as a purposive sampling framework in order to organise and summarise the most promising research in each aspect of prevention. Ideally, the selected framework would be able to accommodate research at different levels of maturity (e.g. from predominantly observational / ecological studies to randomised clinical trial(RCT)-type intervention trials). While there are now several ecological frameworks available for categorising the likely causes of obesity and corresponding targets for intervention, the Analysis Grid for Environments Linked to Obesity (ANGELO) framework from Swinburn et al. [6] has been used specifically to map broad portfolios of interventions in other countries, most notably in Pacific island communities where rates of obesity in adults are among the highest in the world [7].

The ANGELO framework categorizes all societal and environmental drivers (and, by extension, their potential target intervention points) of obesity across the four domains of: physical, economic, legislative and socio-cultural environments. It is then sub-divided into two 'scales': the macro-level denoting factors or policies which operate at the regional or state level and the micro-level'relating to the household, institutional or community scale. In their introductory paper, the developers of the framework outline how 'micro-environ- 
Mooney et al.: Obesity Prevention in Scotland: A Policy Analysis Using the ANGELO Framework

Table 1. Analysis grid for environments linked to obesity (ANGELO) (as adapted from Swinburn et al. [6])

\begin{tabular}{lll}
\hline Domain & Scale & $\begin{array}{l}\text { macro-environment } \\
\text { (e.g. regional; national) } \\
\text { diet-related / activity-related }\end{array}$ \\
\cline { 2 - 3 } & $\begin{array}{l}\text { micro-environment } \\
\text { (e.gousehold; community) }\end{array}$ & $\begin{array}{l}\text { diet-related / activity-related } \\
\text { Physical }\end{array}$ \\
& What is available, for example, buildings, amenities, facilities and use patterns? \\
Economic & What are the monetary cost factors/influences/consequences? Includes price \\
\hline Legislative & incentives / disincentives / taxes and cost savings driven measures \\
\hline Socio-cultural & What are the rules/legal guidance/statutory provisions/political messages? \\
& Also includes codes of conduct and acceptable standard practices / behaviours \\
\hline
\end{tabular}

mental settings' such as schools, homes, workplaces and neighbourhoods are in turn influenced by the broader 'macro-environments' or sectors such education or health systems, all levels of Government, the food industry and a society's attitudes and beliefs [6]. Finally, causal factors or intervention policies are further classified according to whether they are mediated through 'diet' or 'physical activity' (table 1 summarizes the ANGELO categorisation framework).

For the current analysis we considered the 62 policies and programmes of the Scottish Government's Route Map Action Plan from the perspective of the ANGELO framework to assess balance and spread of the proposed and on-going intervention measures according to predominant domain(s), scale and underlying focus (i.e. diet or physical activity).

\section{Material and Methods}

Using the ANGELO introductory paper [6] as guidance, two assessors (JM \& RJ) independently allocated a category for each intervention according to its scale (macro or micro); domain (physical, economic, legislative or sociocultural) and predominant focus (physical activity or diet). A third assessor (RG) then examined those policies for which there were non-matching allocations, and a consensus on each was agreed by discussion between all three. In simple terms, the four domains are:

- $\quad$ Physical: what is available in terms of buildings, structures and amenities?

- Economic: what are the costs / financial measures including incentives, penalties and cost-benefits?

- Legislative: what are the rules and regulations? (i.e. at the national, local or institutional level)

- Socio-cultural: what are the prevailing attitudes and beliefs as well as accepted values and

behaviours?

In reviewing each policy or programme measure, assessors allocated a score of 1.0 for the eventual subcategory into which it was allocated. Where a particular programme clearly had distinct and separate contributions from more than one category (e.g., a new cycle path (physical) introduced alongside a concerted educational and behavioural campaign (socio-cultural)), assessors were asked to assign a value of 0.5 to each category. The increased risk of subjectivity in making further sub-divisions to the 'unit' of intervention led the investigators to restrict the sub-division of points to no more than two sub-categories. At the stage where a consensus had been reached about the allocations of each policy measure, the points totals were tallied across domains and the spread of measures compared with what might be seen as ideally based on the findings of our earlier review which also employed the ANGELO framework [5]. Within the results and discussion section, Action Plan policy options are preceded by the letters AP and italicised, (e.g. Action Plan Component $2.4=$ AP 2.4). 
Mooney et al.: Obesity Prevention in Scotland: A Policy Analysis Using the ANGELO Framework

Table 2. Prevention of Obesity Route-Map Action Plan: categorisation by ANGELO domain

\begin{tabular}{|c|c|c|c|c|c|c|}
\hline \multirow[t]{3}{*}{ Domain } & \multicolumn{4}{|l|}{ Scale } & \multirow{2}{*}{\multicolumn{2}{|c|}{ Domain breakdown }} \\
\hline & \multicolumn{2}{|c|}{ Macro-environment } & \multicolumn{2}{|c|}{ Micro-environment } & & \\
\hline & dietary & activity & dietary & activity & total & $\%$ \\
\hline Physical & 1 & 7.5 & 3 & 8 & 19.5 & 31.5 \\
\hline Economic & 1.5 & 1 & 1.5 & 1 & 5 & 8.1 \\
\hline Legislative & 6.5 & 3 & 3.5 & 1.5 & 14.5 & 23.4 \\
\hline Socio-cultural & 5 & 5.5 & 6 & 6.5 & 23 & 37.1 \\
\hline Total & 14 & 17 & 14 & 17 & & \\
\hline$\%$ of total & 22.6 & 27.4 & 22.6 & 27.4 & & \\
\hline Dietary to activity ratio & & $14: 17$ & & & & \\
\hline Scale total & & 31 & & & & \\
\hline
\end{tabular}

\section{Results}

\section{The Physical Domain}

Table 2 shows the total policy component allocations by ANGELO domain for all current and planned programmes described in the Route-Map Action Plan. Policies and programmes which are orientated towards physical surroundings and amenities have a strong preponderance towards operating through increased opportunities for physical activity, as opposed to dietary measures. Of the total policy score of 19.5 for the physical domain,15.5 (79.5\%) were mediated through activity-based measures, the remaining 4.0 policy 'units' being concerned with improving access to a healthy diet. Among the activity-orientated measures, it is notable that they are evenly split between macro-level and micro-level scale. In contrast, diet-orientated measures within the physical domain are predominantly at the micro-level (8 out of 9) within defined local settings such as schools and workplaces. The need at this level for improved availability to be accompanied by targeted information and skill development meant that both AP 1.8 (working with food outlets around schools) and AP 1.12 (community food co-ops with cookery skills component) [8] were split between the physical and sociocultural domains.

\section{The Economic Domain}

As a whole, interventions which are classified under the economic domain have a relatively low representation at just 5.0 (or $8.1 \%$ ), of policy units out of a total of 62 . Both activityorientated interventions within the economic domain also involve the provision of financial support to external organisations. At the macro-scale, there is a commitment to support the third sector to implement a national 'active-play' programme for children (AP 3.11), and at the micro-level there is targeted support/incentives to encourage workplace travel planning with committed employers (AP 4.9). Since 'Healthy Start' vitamin vouchers (AP 3.4) for eligible pregnant women are both a national policy and individually targeted, they have been splitcategorised between macro-level and micro-level measures. The voucher's coverage was also recently extended to include frozen fruit and vegetables as well as dietary supplements.

\section{The Legislative Domain}

Almost a quarter (14.5 out of 62 or $23.4 \%$ ) of the Scottish Government's policy intentions and plans around obesity prevention, in a four-domain framework, are accounted for by legislative-orientated measures. Much of this effort centres on statutory guidance aimed at 
Mooney et al.: Obesity Prevention in Scotland: A Policy Analysis Using the ANGELO Framework

improving dietary standards for key population sub-groups such as school children (AP 1.6), and infants (APs $3.2 \& 3.10$ ). The package of legislative initiatives also looks towards extending the widely adopted 'healthy living award' for workplaces to the commercial catering sector (AP 1.4), and contains a continuing commitment to the pre-watershed ban on the advertising of foods high in fat, salt and sugar (HFSS) as well as exploring ways to restrict advertising in non-broadcast media ( $A P$ 1.16). The action plan also supports statutory limitations on the numbers of fast-food premises in the vicinity of local schools ( $A P$ 1.7). On the physical activity front, there is recognition at the national planning level of the importance of infrastructure improvement in disadvantaged communities (APS $2.5 \& 2.8$ ), to reduce traffic congestion and promote active travel.

\section{The Socio-Cultural Domain}

The largest category of policy measures documented in the Route-Map Action Plan at nearly $40 \%$ (specifically $23 / 62$ or $37.1 \%$ ), were categorised in the socio-cultural domain of the ANGELO framework. The spread of policies across the four sub-categories of the domain is also the most even, with $6.5 \mathrm{AP}$ points categorised as 'micro-scale/activity and 6.0 as microscale/diet. The equivalent sub-category scores for macro-level policies are 5.5 for activity and 5.0 for diet-related measures (table 2). Furthermore, some of the measures targeted at school dinners, from 'stay in school policies' (AP 1.6(iii)) to examining collaborations with local food outlets $(A P 1.8)$ and tips on healthy affordable lunch box contents $(A P 1.9)$ can be seen within a larger framework of healthier shopping and cooking (AP 1.12). As such, they constitute what might be regarded as a multi-component programme, making maximal use of complimentary 'policy levers' to increase the likelihood of the desired outcome. The same rationale underlies the 'Healthy Working Lives' awards (AP 4.2), the most advanced level of which (i.e. 'Gold award') recognises the need for a comprehensive approach to employee wellbeing.

At the macro-scale level for activity promotion, the panel of assessors agreed that it was important to recognise the importance of cultural attitudes and issues around self-efficacy when considering measures to increase active travel leading to the split categorisations for AP 2.2 (Cycling Action Plan for Scotland: [9]) and AP 2.4 (aimed at reducing journeys delayed by traffic congestion) between the physical and socio-cultural domains. Micro-scale interventions within the socio-cultural domain to promote physical activity are well documented with the Action Plan (e.g. AP 2.14(ii): publicising route information and AP 2.15: targeted active travel planning with higher risk / harder to reach groups). These measures also have several identifiable 'companion policies' from other ANGELO domains without which they are unlikely to be as effective. Measures AP 2.14(i) (expansion of safe routes to link community destinations) under physical environment and $A P 2.16$ (promoting healthy environments for children in every community) are two such examples. Other documented macro-level policies around diet could be said to be relatively non-specific and merely aspirational, e.g. AP 1.14: influencing social norms about food choices and $A P$ 1.15: general awareness raising around what constitutes an energy dense diet.

\section{Overall Categorisation by Scale and Intervention Focus}

When the domain structure is put to one side and overall macro-scale interventions are tallied alongside overall micro-scale interventions, one finding that affords some reassurance as to the overall balance of the entire programme is that 31 , or exactly half of the 62 proposed measures are at the macro-scale level and the other half operate at the micro-scale of intervention. Additionally, within each set of 31, the total numbers of diet-orientated policy measures and the total numbers of physical activity-orientated policies correspond exactly at 14 and 17 respectively. Of course these striking parallels are unlikely to have arisen by deliberate design, since the package of measures as a whole has come together over a number 
Mooney et al.: Obesity Prevention in Scotland: A Policy Analysis Using the ANGELO Framework

of years with different policies at different stages of development and having different origins. From the perspective of the overall policy mix however, the distribution is not heavily skewed in terms of either scale of intervention or predominant focus (i.e. activity or diet).

\section{Discussion}

The ANGELO framework has previously been used to prioritise obesity prevention policies in four Pacific countries in the 'Obesity Prevention in Communities' (OPIC) project [7]. Within OPIC, it helped to identify and categorise potential interventions ahead of implementation and was an effective means of engaging community-level stakeholders in tailoring programmes that were locally relevant and responsive to their respective 'stage of readiness'. Given that the Scottish Government had already drawn together a set of policies and programmes that could potentially contribute to obesity prevention, our own starting point was to use ANGELO to examine the balance of existing measures, with a view to highlighting any obvious gaps or imbalances. Our discussion presents an overview of the policy analysis across the four domain structures of ANGELO and highlights potential implications for policy implementation from both a Scottish and an international perspective.

Given the capital investment levels which are normally required to improve the built environment for the purposes of active travel [9], the predominance of activity-based measures (over those based on diet) within the physical domain might be seen as initially surprising. It is now relatively well established however that environmental improvements (such as expansion of the national cycle network (AP 2.2), are likely to be of limited benefit on new habit formation, without also tackling the wider environmental determinants such as 'safety perception' (lighting and local levels of disorder) [10] (AP 2.14(iii)) and the accessibility of good sports facilities in school and community locations (AP 2.17).

Provisions within national planning guidance for infrastructure improvements to assist active travel were split allocated to both physical and legislative domains. The two action plan items, AP 2.5 and 2.8 are underpinned by the national planning framework for Scotland [11] and the policy statement, 'Designing Streets' [12] respectively. The former sets out a series of specific objectives around improving sustainable transport infrastructure and the latter advocates a 'place-based' approach to street design and re-development with a focus on residents and users. The challenges of evaluating place-based approaches in particular have been well documented in non-UK settings such as in Canada [13], where their evolving nature and appropriate measure selection have presented difficulties. This highlights the need for welldefined pilot areas if such approaches were to be trialled in Scotland.

For macro-level economic measures, there is now a clear international consensus that marginal price increases could be an effective disincentive to purchasing high-energy minimally nutritious food and soft drinks [14]. With sugared beverages for example, large-scale longitudinal surveys as well as price sensitivity analyses have highlighted reduced consumption after price increases in high-risk groups alongside the expected attendant health benefits [15]. The economic importance and lobbying power of the international food industry however means that most governments are inclined to avoid supplementary taxation. There are also additional logistical difficulties around challenges to trade and the extent to which regional administrations have the necessary authority to act, especially with international free-trade agreements such as those within the EU or the Americas [16]. Given the above challenges, the restriction of the one macro-level policy programme that is fully allocated to the economic/dietary category to 'financial support for industry to assist with reformulation' ( $A P 1.2$ ) is perhaps understandable. It is also worth highlighting however that reformulation is an area where there has been a track record of moderate success already in 
Mooney et al.: Obesity Prevention in Scotland: A Policy Analysis Using the ANGELO Framework

the UK with reductions in salt content [17]. At the micro-economic level, however, there is a disappointing lack of any guidance or support for micro-level economic incentives for institutional settings such as schools and workplaces, which is well evidenced from international studies $[18,19]$. Such micro-level measures are also most effective when implemented in combination with supportive measures around knowledge and skills as demonstrated in community-based projects [8].

In contrast with the seeming reluctance to embrace economic measures at the institutional level, both the Scottish and UK Governments have demonstrated a greater enthusiasm for the legislative domain around guidance for both activity and nutritional standards. For example, the Scottish Government previously stipulated that by 2014 there should be a minimum of two hours of physical education per week for lower high-school pupils (S1-S4) (AP 2.18). Left to local implementation plans at the school level (and therefore defined as micro-scale), there have been significant reservations expressed about the adequacy of this standard in isolation, due to the risk of exacerbating the already notable gulf between the activity opportunities in the private school sector and the state [20]. Nutritional standards in schools guidance [21] has in contrast been followed up by complimentary policies and initiatives to encourage pupils to stay on-site during lunch time [22] (AP 1.6(iii): socio-cultural) and statutory measures to tackle the easy availability of fast-foods around schools (AP 1.7: legislative). Given that fast-food outlets have been noted to cluster near school locations in cities in the UK and internationally $[23,24]$, councils could in principle be empowered to restrict licensing, an option which has mainly been investigated in London; a local government guide is now available [25].

Mass media advertising is clearly identifiable as a macro-level component of the sociocultural domain, and although broadcasting restrictions at the UK level had been found to reduce the broadcasting of HFSS adverts during children's programming times, the industry has been able to exploit alternative scheduling (for example to family viewing times), to maintain exposure levels [26]. Also, given the acknowledged importance of food consumed outside the home, high street promotions (e.g. of fast-food meals) to school children ought to be an important area of focus [27]. Also allocated within the socio-cultural domain, policy measures to improve diet based on information and awareness raising, even if appropriately targeted (APS 1.14 \& 1.15), are not likely to be effective [28]. If combined, however, with other action plan policies, such as a statutory approach to food labelling (AP 1.11) (where the traffic light format is preferred [29] or subsidies to encourage healthier product lines (e.g. AP 1.2), there is likely to be a greater chance of starting to address some of the most damaging ingrained cultural barriers to healthy diets in Scotland.

Finally, the slight excess in activity-related measures over dietary measures for both scales of intervention (17 versus 14 for both macro-level and micro-level policies) serves as a reminder that activity- or exercise-orientated interventions are often easier to implement or promote since they generally do not pose a challenge to commercial interests such as food companies or retailers. Indeed, support or sponsorship of sporting events or health campaigns is often seen as a diversion tactic by food and beverage companies, the 2012 London Olympics sponsorship deals [30] and the UK Governments Change4Life campaign [31] being two highprofile examples. While the benefits of increased physical activity for health have never seriously been disputed, it is now widely acknowledged that it is only by tackling the excess availability of cheap high energy-dense foods that obesity epidemic will be curtailed [32]. Rather than advocate an entirely balanced approach between energy in versus energy out, therefore, there is a strong case to be made for more policy efforts on the nutrition side of the equation. At the very least, this should keep a check on large-scale diversionary tactics by big food companies and serve as a prompt to governments not to retreat from the more challenging (and likely to be more effective), anti-obesity programmes and policies. 
Mooney et al.: Obesity Prevention in Scotland: A Policy Analysis Using the ANGELO Framework

\section{Conclusion}

The Scottish Government's Route-Map Action Plan is one of the first attempts to set out at a national level a broad multicomponent package of genuinely cross-government measures to tackle obesity. Analysis of the plan as a whole using the ANGELO framework for policy interventions to tackle obesity demonstrates that, while all of the four 'domains' of physical, economic, legislative and socio-cultural influences are represented, there is a disproportionate imbalance of policies in the attitude / behavioural arena and to a lesser extent for the built environment, at the expense of the legislative and particularly economic domains. This picture is unsurprising and is likely to be typical for most governments or national administrations which are subject to strong international trade agreements and other factors that are not entirely within their own control. However, it is also at odds with the increasing body of international evidence around what works best. Should policy makers therefore be genuinely looking to address the obesogenic environment, they ought to be encouraged to strive for a more evenly balanced approach, with greater emphasis on both fiscal and legislative levers while at the same time countering any natural tendency towards a preference for exercise-based interventions in favour of directly addressing the oversupply of cheap high energy-dense foods. The ANGELO grid and the above type of policy analysis are a convenient and appropriate framework to assess and monitor efforts over time to achieve this particular balance of emphasis.

The Scottish Government has to date demonstrated considerable political courage and innovative flair in the public health policy arena, being one of the first jurisdictions to successfully introduce a smoking ban in public places [33] and more recently by its continued commitment to the implementation of an alcohol minimum unit pricing policy [34]. Addressing some practices of the food industry, by seriously considering macro-level policies which would impact on diet, specifically around targeting the low cost and easy accessibility of energy-dense, nutritionally poor food choices, would be a fitting bold next step. Such a move would also constitute a more genuinely comprehensive policy approach to tackling obesity prevention at the population level, around the need for which there is now a clear universal consensus.

\section{Acknowledgement}

We would like to gratefully acknowledge the contribution of Professor Geraldine McNeill, Chair in Public Health Nutrition at the University of Aberdeen, for her very helpful comments on the draft manuscript.

\section{Disclosure Statement}

All authors declare that we have no conflicts of interest, commercial or otherwise, in relation to the work presented.

\section{References}

1 Scottish Public Health Observatory (ScotPHO): Obesity in Scotland: An Epidemiology Briefing. Edinburgh, 2007.

2 Scottish Government: Preventing Overweight and Obesity in Scotland: A Route Map towards A Healthy Weight. Edinburgh, 2010.

3 Foresight: Tackling Obesities: Future Choices, 2nd ed. London, Government Office for Science. 2009.

4 Scottish Government: Prevention of Obesity Route Map - Action Plan 1.1. Edinburgh, 2011.

5 Mooney JD, Frank J, Haw S: Policy Interventions to Tackle the Obesogenic Environment - Focusing on Adults of Working Age in Scotland. Edinburgh, Scottish Collaboration for Public Health Research and Policy. 2011. 
Mooney et al.: Obesity Prevention in Scotland: A Policy Analysis Using the ANGELO Framework

6 Swinburn B, Egger G, Raza F: Dissecting obesogenic environments: the development and application of a framework for identifying and prioritizing environmental interventions for obesity. Prev Med 1999;29:563570.

7 Schultz J, Utter J, Mathews L, Cama T, Mavoa H, Swinburn B: The Pacific OPIC Project (Obesity Prevention in Communities): action plans and interventions. Pac Health Dialog 2007;14:147-153.

8 Rees R, Hinds K, Dickson K, O’Mara-Eves A, Thomas J: Communities that Cook: A Systematic Review of the Effectiveness and Appropriateness of Interventions to Introduce Adults to Home Cooking. London, Institute of Education, University of London, EPPI-Centre, 2012.

9 Transport Scotland: Cycling Action Plan for Scotland. Edinburgh, 2013.

10 Ogilvie D, Mitchell R, Mutrie N, Petticrew M, Platt S: Personal and environmental correlates of active travel and physical activity in a deprived urban population. Int J Behav Nutr Phys Act 2008;5:43.

11 Scottish Government: National Planning Framework 3. Edinburgh, 2014.

12 Scottish Government: Designing Streets: A Policy Statement for Scotland. Edinburgh, 2010.

13 Bellefontaine T, Wisener R: The Evaluation of Place-Based Approaches: Questions for Further Research. Toronto, Policy Horizons Canada, 2011.

14 Brownell KD, Frieden TR: Ounces of prevention - the public policy case for taxes on sugared beverages. N Engl J Med 2009;360:1805-1808.

15 Sturm R, Datar A: Body mass index in elementary school children, metropolitan area food prices and food outlet density. Public Health 2005;119(1059-1068.

16 Labonte R, Mohindra KS, Lencucha R: Framing international trade and chronic disease. Global Health 2011;7: 21.

17 He FJ, MacGregor GA: A comprehensive review on salt and health and current experience of worldwide salt reduction programmes. J Hum Hypertens 2009; 23:363-384.

18 French S, Jeffery R, Story M, Hannan P, Snyder M: A pricing strategy to promote low fat snack choices through vending machines. Am J Public Health 1997;87:849-851.

19 French SA, Harnack LJ, Hannan PJ, Mitchell NR, Gerlach AF, Toomey TL: Worksite environment intervention to prevent obesity among metropolitan transit workers. Prev Med 2010;50:180-185.

20 Davies EJ, Edwards KL: A pilot study comparing the physical activity levels of children at independent and state schools in Sheffield, United Kingdom. Obes Facts 2013;6(suppl 1):202.

21 Scottish Government: Nutrition in Schools: Scottish Nutrient Standards for School Lunches. Edinburgh, 2003.

22 Glasgow Centre for Population Health: Evaluating the Impact of the 'Big Eat In' Secondary School Pilot. Findings Series 27 Briefing Paper. Glasgow, 2011 www.gcph.co.uk/publications/226_findings_series_27-the_ big_eat_in (last accessed July 13, 2015).

23 Davis B, Carpenter C: Proximity of fast-food restaurants to schools and adolescent obesity. Am J Public Health 2009;99:505-510.

24 Ellaway A, Macdonald L, Lamb K, Thornton L, Day P, Pearce J: Do obesity-promoting food environments cluster around socially disadvantaged schools in Glasgow, Scotland? Health Place 2012;18:1335-1340.

25 Greater London Authority Council: Takeaways Toolkit: Tools, Interventions and Case Studies to Help Local Authorities Develop a Response to the Health Impacts of Fast Food Takeaways. London, 2012.

-26 Adams J, Tyrrell R, Adamson AJ, White M: Effect of restrictions on television food advertising to children on exposure to advertisements for 'less healthy' foods: repeat cross-sectional study. PLoS ONE 2012; 7:e3157827.

27 Cairns G, Angus K, Hastings G, Caraher M: Systematic reviews of the evidence on the nature, extent and effects of food marketing to children. A retrospective summary. Appetite 2013;62:209-215.

28 Ni Mhurchu C, Aston L, Jebb S: Effects of worksite health promotion interventions on employee diets: a systematic review. BMC Public Health 2010;10:62.

29 Borgmeier I, Westenhoefer J: Impact of different food label formats on healthiness evaluation and food choice of consumers: a randomized-controlled study. BMC Public Health 2009;9:184.

30 Clark M, Brownell R: The Obesity Games: the Inside Track on the Marketing Strategies of Olympic Food and Soft Drink Sponsors, and the Sponsorship Deals Behind Them. London, The Children's Food Campaign, 2012. Editorial: Change4Life brought to you by PepsiCo (and others). Lancet 2009;373:96.

31 Swinburn BA, Sacks G, Hall KD, McPherson K, Finegood DT, Moodie ML, Gortmaker SL: The global obesity pandemic: shaped by global drivers and local environments. Lancet 2011;378:804-814.

-33 Pell JP, Haw S, Cobbe S, Newby DE, Pell AC, Fischbacher C, McConnachie A, Pringle S, Murdoch D, Dunn F, Oldroyd K, Macintyre P, O’Rourke B, Borland W: Smoke-free legislation and hospitalizations for acute coronary syndrome. N Engl J Med 2008;359:482-491.

34 Scottish Government: Minimum Unit Pricing for Alcohol. www.gov.scot/Topics/Health/Services/Alcohol/ minimum-pricing (last accessed July 13, 2015). 